\title{
BMJ Open Do people living in rural and urban locations experience differences in harm when admitted to hospital? A cross- sectional New Zealand general practice records review study
}

\author{
Carol Atmore (10 , ${ }^{1}$ Susan Dovey, ${ }^{1}$ Robin Gauld (10 , ${ }^{2}$ Andrew R Gray (1) , ${ }^{3}$ \\ Tim Stokes (iD) ${ }^{1}$
}

To cite: Atmore C, Dovey S, Gauld R, et al. Do people living in rural and urban locations experience differences in harm when admitted to hospital? A cross-sectional New Zealand general practice records review study. BMJ Open 2021;11:e046207. doi:10.1136/ bmjopen-2020-046207

- Prepublication history for this paper is available online. To view these files, please visit the journal online (http://dx.doi. org/10.1136/bmjopen-2020046207).

Received 23 October 2020

Revised 01 April 2021

Accepted 16 April 2021

D) Check for updates

(c) Author(s) (or their employer(s)) 2021. Re-use permitted under CC BY-NC. No commercial re-use. See rights and permissions. Published by BMJ.

${ }^{1}$ Department of General Practice and Rural Health, Dunedin School of Medicine, Otago Medical School, University of Otago, Dunedin, New Zealand 2Dean's Office, Otago Business School, University of Otago, Dunedin, New Zealand ${ }^{3}$ Biostatistics Centre, Division of Health Sciences, University of Otago, Dunedin, New Zealand

Correspondence to

Dr Carol Atmore;

carol.atmore@otago.ac.nz

\section{ABSTRACT}

Objective Little is known about differences in hospital harm (injury, suffering, disability, disease or death arising from hospital care) when people from rural and urban locations require hospital care. This study aimed to assess whether hospital harm risk differed by patients' rural or urban location using general practice data.

Design Secondary analysis of a 3-year retrospective cross-sectional general practice records review study, designed with equal numbers of rural and urban patients and patients from small, medium and large practices. Hospital admissions, interhospital transfer and hospital harm were identified.

Setting New Zealand (NZ) general practice clinical records including hospital discharge data.

Participants Randomly selected patient records from randomly selected general practices across NZ. Patient enrolment at rural and urban general practices defined patient location.

Outcomes Admission and harm risk and rate ratios by rural-urban location were investigated using multivariable analyses adjusted for age, sex, ethnicity, deprivation, practice size. Preventable hospital harm, harm severity and harm associated with interhospital transfer were analysed.

Results 0 of 9076 patient records, 1561 patients (17\%) experienced hospital admissions with no significant association between patient location and hospital admission (rural vs urban adjusted risk ratio (aRR) 0.98 (95\% Cl 0.83 to 1.17)). Of patients admitted to hospital, $172(11 \%)$ experienced hospital harm. Rural location was not associated with increased hospital harm risk (aRR 1.01 (95\% Cl 0.97 to 1.05)) or rate of hospital harm per admission (adjusted incidence rate ratio 1.09 (95\% $\mathrm{Cl} 0.83$ to 1.43$)$ ). Nearly half (45\%) of hospital harms became apparent only after discharge. No urban patients required interhospital transfer, but $3 \%$ of rural patients did. Interhospital transfer was associated with over twice the risk of hospital harm (age-adjusted aRR 2.33 (95\% Cl 1.37 to 3.98), $p=0.003$ ).

Conclusions Rural patient location was not associated with increased hospital harm. This provides reassurance for rural communities and health planners. The exception
Strengths and limitations of this study

- Secondary analysis of a large retrospective patient record review study has allowed us to investigate associations of clinical significance between patients' rural and urban location and hospital harm.

- The selection of similar numbers of patient records from rural and urban general practices in the study design effectively maximised the study's statistical power to detect urban-rural differences.

- Our study examined data from general practice electronic patient records, which provided the opportunity to detect harms not identified in hospitalonly patient record review studies.

- Defining patients' rural or urban location by their general practice location may have miscategorised some rural patients as urban.

- Data on patient health or illness status were not collected and are likely to be residual confounders.

was patients needing interhospital transfer, where risk was more than doubled, warranting further research.

\section{INTRODUCTION}

In many European and North American countries, at least one-third of the population live in rural regions. ${ }^{1}$ Using Statistics New Zealand's definition of 'rural' (living in communities of 999 people or fewer), $16 \%$ of the New Zealand (NZ) population is classified as rural, and a similar percentage of the NZ population lives in town of 30,000 people or less, distant to major population centres. ${ }^{2}$ The ability to access good quality hospital care when required is of major concern to people living in small towns and rural communities (rural people) and healthcare planners. ${ }^{3-6}$ Rural people receive hospital care at their local smaller rural hospitals (where present) and larger urban hospitals. Some people 
require transfer between hospital sites during hospital admission. Little, however, is known about the quality of hospital services rural people receive compared with their urban-dwelling neighbours.

The American Institute of Medicine identifies patient safety is one aspect of healthcare quality. ${ }^{7}$ Research into patient safety differences in hospital care by location is limited and relates to the rural-urban location of hospitals rather than where people live. No appreciable difference has been found in patient safety ${ }^{8}$ and adverse events ${ }^{9}$ in similar-sized American rural and urban hospitals, but international studies show that interhospital patient transfer is associated with adverse outcomes. ${ }^{10-14}$ These include including delay in time to surgery, ${ }^{10}$ longer length of hospital stay, ${ }^{11} 12$ longer time in intensive care unit $^{12}$ and higher inpatient mortality compared with patients not transferred during admission. ${ }^{13}$

Adverse events (incidents that results in patient harm) related to hospital admissions prolong hospital stays and cause additional hospital costs, disability and death. ${ }^{15-18}$ Most hospital patient safety studies collect data through hospital clinical notes review, and identify adverse events or the resulting harms (injury, suffering, disability, disease or death arising from healthcare $)^{18}$ during the index or subsequent hospital admissions. ${ }^{15-17}$ Harms that become evident after discharge but do not require hospital readmission will be missed. The proportion of these undetected harms seen only in primary care settings is not known.

To explore if differences in hospital-related safety outcome existed by rural-urban place of patient residence, we investigated whether there were differences in harm experienced by people admitted to hospital who attended general practices in rural and urban NZ settings.

\section{METHODS}

\section{Study design and data collection}

Differences in hospital harm experienced by rural and urban patients were investigated through secondary analysis of the 'Safety, Harms and Risk reduction Project' (SHARP) study data, ${ }^{19}$ a retrospective cross-sectional general practice patient records review study. This preexisting dataset comprised electronic clinical records of 9076 randomly selected patients from 44 randomly selected large (4500 or more patients), medium (20004499 patients) and small ( $<2000$ patients) general practices throughout NZ, covering the three calendar years of 2011-2013. We used patient attendance at rural and urban general practices as a proxy for place of residence.

Stratification by both location and size ensured that similar numbers of patients were included from rural and urban general practices. Participating practices used the same computerised patient records software, estimated to be used by $80 \%$ of NZ practices. ${ }^{19}$ Patient records included hospital discharge and outpatient attendance summaries, as well as full records of general practice encounters. Records had been reviewed in the original SHARP study and all harms, defined by the researchers as "physical, emotional or financial negative consequences to patients directly arising from healthcare, beyond the usual consequences of care and not attributable to patients' health condition', had been identified. ${ }^{19}$

For this study, one author (CA) manually reviewed the SHARP dataset of 9076 patient records to identify hospital admissions, including interhospital transfers. The author focused on communications between hospitals, medical specialists and general practices to identify admissions. The author also reviewed the 2999 harms noted in the SHARP study, and identified 195 patient records where harms had resulted from hospital admissions (hospital harms). In 23 of the 195 (12\%) patient records with hospital harm, the author was unable to identify a contemporaneous hospital admission. In 10 patient records, there was no documentation from the hospital to indicate hospital admission had occurred. In 13 patient records, the admission record was not detected through the admission identification process described. The 172 patient records where the author had identified the hospital admission associated with the hospital harm were compared with the 23 patient records where no corresponding hospital admission had been identified. There was no difference in rural or urban status $(\mathrm{p}=0.573)$. The patients with no corresponding hospital admission identified were younger (median age 47 vs 64 years, $p=0.005$ ) and more likely to be female $(73.9 \%$ vs $45.6 \%, \mathrm{p}=0.045)$, but no significant differences in ethnicity, sociodemographic status or practice size were seen. These 23 patient records were excluded from further analysis.

The inter-rater reliability ${ }^{20-22}$ of the original SHARP study data showed moderate agreement for the presence of hospital harm (kappa statistic 0.40 (95\% CI 0.31 to $0.49)$ ), but poor agreement for number of hospital harms detected (weighted kappa statistic 0.30 (95\% CI 0.23 to $0.38)$ ). Therefore, we categorised our data into hospital admissions that resulted in any, or no, harm. For hospital harms identified by two SHARP researchers, the preventability and severity codes assigned (see table 1) showed substantial agreement (kappa statistic for preventability $=0.73$ (95\% CI 0.34 to 1.00$)$; weighted kappa statistic for severity 0.64 (95\% CI 0.27 to 0.89 )).

Our dataset of patient records included general practice and hospital information. As a secondary objective, we explored where in the patient journey hospital harm was identified. Hospital discharge summaries and closely time-proximate general practice consultations were examined to determine if hospital harms were identified during initial or subsequent hospital admissions. Harms were classified as detected only in general practice if the patient records indicated that hospital harms became apparent only after discharge and were not associated with readmission.

Definitions used during data collection and analysis are described in table 1, including the definition of rural used in the original SHARP study. ${ }^{23}{ }^{24}$ For ethnicity, $2 \%$ of records had no ethnicity information and were included 


\begin{tabular}{lc}
\hline Table 1 & Data definitions \\
\hline Variable & Definition \\
\hline Outcomes & \\
$\begin{array}{l}\text { Hospital } \\
\text { admission }\end{array}$ & $\begin{array}{l}\text { An identified hospital admission, which could include } \\
\text { interhospital transfer between hospitals in different } \\
\text { towns or cities as part of the same inpatient hospital } \\
\text { experience. }\end{array}$
\end{tabular}

Hospital harm Patient harm as a result of hospital admission.

Where harm Setting where hospital harm detected, either during detected hospital admissions, or in general practice only.

$\begin{array}{ll}\begin{array}{l}\text { Preventable } \\ \text { harm }\end{array} & \begin{array}{l}\text { Any hospital harm experienced by the patient and } \\ \text { coded as 'preventable and originating in secondary } \\ \text { care' by SHARP researchers. }\end{array}{ }^{19}\end{array}$

Harm severity The highest severity coding of any identified hospital harm experienced by a unique patient using the three codes of minor, moderate and severe (including death) allocated by SHARP researchers. ${ }^{19}$

$\begin{array}{ll}\text { Exposure } & \text { The rural or urban location of general practice } \\ \text { attended by patients, as a proxy for patient } \\ \text { residence. } \\ \text { Rural: patients attending general practices with } \\ \text { addresses in rural centres of }<1000 \text { inhabitants or } \\ \text { independent urban areas of }<30000 \text { inhabitants with } \\ \text { little major urban centre influence, as defined by } \\ \text { Statistics NZ. } \\ \text { Urban: patients attending all other general practices. }\end{array}$
Covariates

$\begin{array}{ll}\text { Age } & \text { In years at mid point of data collection (1 July 2012). } \\ \text { Sex } & \text { As recorded in the patient record. }\end{array}$

Ethnicity Māori, Pacific, 'other', then European, as recorded in the patient record, using prioritised Statistics NZ definitions and level 1 codes, and grouped and prioritised in above order. ${ }^{42}$ 'Other' included all other ethnicities not named above, and missing ethnicity data.

$\begin{array}{ll}\text { Deprivation } & \begin{array}{l}\text { Six categories: quintiles } 1 \text { (least deprived) to } 5 \text { (most } \\ \text { deprived), derived from the NZDep13 quintile } \\ \text { the recorded patient address in the patient record, } \\ \text { linked through address geo-coding, }{ }^{29} \text { with a sixth } \\ \text { category to record missing deprivation data. }\end{array} \\ \text { Practice size } & \begin{array}{l}\text { Defined by tertiles of NZ general practices by } \\ \text { number of enrolled patients as per SHARP } \\ \text { researchers. }\end{array} \\ & \text { Large: } 4500 \text { or more patients, mean } 7893 \text { enrolled } \\ & \text { patients per practice, minimum-maximum } 4512- \\ & 16682 \text { patients. } \\ & \text { Medium: } 2000-4499 \text { patients, mean } 3117 \text { enrolled } \\ & \text { patients per practice, minimum-maximum } 2342- \\ & 4355 \text { patients. } \\ \text { Small: }<2000 \text { patients, mean } 1420 \text { enrolled patients } \\ \text { per practice, minimum-maximum } 660-1992 \text { patients. }\end{array}$

NZ, New Zealand; SHARP, Safety, Harms and Risk reduction Project.

in the 'other' category. While 'other' ethnicity was already uninterpretable as a group due to its heterogeneity, this approach still allowed for interpretable comparisons between people of Māori, Pacific and European ethnicities. For deprivation, a separate category for missing data was included as $10 \%$ of patient records were missing this information, with similar limitations on interpretation.

\section{Data analysis}

Data were described and analysed to investigate whether patients attending rural general practices had different risks of hospital admission, hospital harm or different patterns of hospital harm severity or preventability compared with patients attending urban general practices. The association between where hospital harm was detected and location was described. Hospital harm was analysed relating to interhospital transfer.

Descriptive statistics were presented as means and SD for normally distributed continuous variables, medians and 25th and 75th percentiles for other continuous variables, and counts and percentages for categorical variables. Associations between categorical variables were examined using $\chi^{2}$ tests (or Fisher's exact test if expected cell counts were below 5 in $>20 \%$ of cells), and between continuous and categorical variables using independent-sample t-tests (when residuals were normally distributed) or non-parametric Mann-Whitney $\mathrm{U}$ tests (otherwise). Poisson regression (as no evidence of overdispersion was present) and ordinal logistic regression models were used to produce relative risk and incidence rate ratios, and proportional ORs, respectively. Potential confounders of associations were controlled by including age (per year increment), sex, ethnicity, deprivation and general practice size in models, except where stated otherwise. Evidence of non-linearity for the continuous variable age was explored using quadratic and cubic terms $\left(\mathrm{age}^{2}\right.$ and age $^{3}$ ), and potential interactions between location and other variables were assessed in the regression models using Wald tests, and retained only if statistically significant. Collinearity was examined by looking at variance inflation factors, where values of $<5$ were considered to indicate a lack of issue.

Clustering of patients within participating general practices was taken into account using clustered robust SEs. ${ }^{25}$ Weights developed from the SHARP study data were applied to enable generalisability of results to the NZ population.

As this study was secondary analysis, no formal sample size calculations were performed. We considered the likely number of events sufficient to allow for the most complex modelling anticipated for exploring admission and hospital harm differences by rural/urban location. ${ }^{26}$ The retrospective power of the analysis as performed was communicated through the widths of reported CIs. Analyses were undertaken using the Stata-IC V.15.1 statistical analysis package. ${ }^{27}$ Results were considered statistically significant when two-sided $\mathrm{p}$ values were $<0.050$. Associated $95 \%$ CIs were presented to aid in interpreting potential clinical significance.

\section{Patient and public involvement}

Patients and/or the public were not involved in the design, conduct, reporting, or dissemination plans of this research.

\section{RESULTS}

\section{Patient characteristics}

Patient characteristics for the whole study group, patients with hospital admissions and with hospital harm, by rural 
Table 2 Patient characteristics

\begin{tabular}{|c|c|c|c|c|c|c|c|}
\hline \multirow[b]{2}{*}{ Variables } & & \multicolumn{2}{|c|}{ Whole study group $n=9076$} & \multicolumn{2}{|c|}{$\begin{array}{l}\text { Patients with hospital } \\
\text { admissions } n=1561\end{array}$} & \multicolumn{2}{|c|}{$\begin{array}{l}\text { Patients with hospital } \\
\text { harm } n=172\end{array}$} \\
\hline & & $\begin{array}{l}\text { Urban } \\
n=4544 n(\%) \dagger\end{array}$ & $\begin{array}{l}\text { Rural } \\
n=4532 n(\%) \dagger\end{array}$ & $\begin{array}{l}\text { Urban } n=752 n \\
(\%) \dagger\end{array}$ & $\begin{array}{l}\text { Rural } \\
n=809 n(\%) \dagger\end{array}$ & $\begin{array}{l}\text { Urban } n=79 n \\
(\%) \dagger\end{array}$ & $\begin{array}{l}\text { Rural } \\
n=93 n(\%) \dagger\end{array}$ \\
\hline \multirow[t]{3}{*}{ Practice size } & Large & $1501(33)$ & $1502(33)$ & $263(35)$ & $244(30)$ & $28(47)$ & $32(54)$ \\
\hline & Medium & 1543 (34) & 1537 (34) & $274(36)$ & 287 (36) & 27 (44) & $35(56)$ \\
\hline & Small & 1500 (33) & 1493 (33) & 215 (29) & $278(34)^{*}$ & $24(48)$ & $26(52)$ \\
\hline \multirow[t]{2}{*}{ Sex } & Male & 2078 (46) & $2226(49)^{\star \star}$ & $332(44)$ & $386(48)$ & $35(44)$ & $48(52)$ \\
\hline & Female & 2466 (54) & $2306(51)$ & $420(56)$ & $423(52)$ & $44(56)$ & $45(48)$ \\
\hline \multirow[t]{2}{*}{ Age (years) } & Mean (SD) & $41(24)$ & $42(24)$ & $53(25)$ & $54(24)$ & $62(19)$ & $59(20)$ \\
\hline & $\begin{array}{l}\text { Median } \\
\text { (25th-75th } \\
\text { percentile) }\end{array}$ & $42(21-59)$ & $44(20-61)$ & $56(36-74)$ & $58(39-72)$ & $66(49-77)$ & $61(50-75)$ \\
\hline \multirow[t]{4}{*}{ Ethnicity $\ddagger$} & European & $3389(75)$ & $3500(77)^{\star \star}$ & $580(77)$ & $650(80)$ & $65(82)$ & $75(81)$ \\
\hline & Māori & $564(12)$ & $762(17)^{\star \star \star}$ & 88 (12) & $121(15)$ & $5(6)$ & $13(14)$ \\
\hline & Pacific & $225(6)$ & $61(1)^{\star \star \star}$ & $52(7)$ & $9(1)^{\star \star \star}$ & 7 (9) & $0(0)^{\star \star}$ \\
\hline & Other & $336(7)$ & $209(5)^{\star \star \star}$ & $32(4)$ & $29(4)$ & $2(3)$ & $5(5)$ \\
\hline \multirow{6}{*}{$\begin{array}{l}\text { Socioeconomic } \\
\text { status-NZDep }\end{array}$} & Quintile 1 & 1040 (23) & $926(20)^{\star \star}$ & $166(22)$ & $149(18)$ & $14(18)$ & $13(14)$ \\
\hline & Quintile 2 & $969(21)$ & $893(20)$ & $163(22)$ & $158(20)$ & 15 (19) & $19(20)$ \\
\hline & Quintile 3 & 868 (19) & $833(18)$ & 140 (19) & $152(19)$ & $14(18)$ & $16(17)$ \\
\hline & Quintile 4 & $693(15)$ & $661(15)$ & 119 (16) & 132 (16) & $13(17)$ & $13(14)$ \\
\hline & Quintile 5 & $664(15)$ & 635 (14) & $113(15)$ & $118(15)$ & $16(20)$ & $16(17)$ \\
\hline & No data & $310(7)$ & $584(13)^{\star \star \star}$ & $51(7)$ & $100(12)^{\star \star \star}$ & $7(9)$ & $16(17)$ \\
\hline
\end{tabular}

${ }^{*} \mathrm{P}<0.05,{ }^{* *} \mathrm{p}<0.01,{ }^{* * *} \mathrm{p}<0.001$.

†Within variable column percentage, except for age data.

$\ddagger$ For ethnicity data, each ethnic group was compared with all other ethnic groups combined, using multiple $\chi^{2}$ tests.

NZ, New Zealand.

and urban location, are shown in table 2. The median age for the whole study group was 43 years (25th-75th percentile 20-60 years), for patients with hospital admissions 57 years (37-73 years), and patients experiencing hospital harm 64 years (49-75 years).

Rural patients represented $50 \%$ of the study group of 9076 patients by design, 52\% of 1561 patients with hospital admissions and $54 \%$ of 172 patients with hospital harm. Fewer patients from large rural practices $(30 \%$ vs $35 \%, \mathrm{p}=0.042)$ and more from small rural practices $(34 \%$ vs $29 \%, \mathrm{p}=0.014)$ experienced hospital admissions than patients attending similar-sized urban practices.

Proportionally more rural patients were male than urban patients ( $49 \%$ vs $46 \%, \mathrm{p}=0.001)$. Fewer rural patients were aged $20-39$ years $(20 \%$ vs $23 \%, \mathrm{p}=0.003)$, and more were aged $60-79$ years $(22 \%$ vs $19 \%, \mathrm{p}=0.001)$. Differences in ethnic distributions in the rural and urban study groups reflected the NZ population. Fewer rural patients in the study group lived in the least deprived areas and $10 \%$ of patient records had no socioeconomic data recorded. Nearly twice as many rural than urban patients had no data on deprivation. Otherwise, the distribution of patient characteristics were similar in rural and urban groups.

\section{Hospital admissions and hospital harms}

Of the study group, 1561/9076 patients (17\%) required hospital admissions during the 3-year study period, of whom 172/1561 patients (11\%) experienced hospital harm. Most patients requiring hospital admissions had one (1052/1561 patients, 67\%) or two (286/1561 patients, $18 \%$ ) admissions over the 3-year study period with no evidence of a difference by location $(\mathrm{p}=0.156)$.

No difference was seen in unadjusted risk of hospital admission for rural compared with urban patients, or risk adjusted for practice size, age, sex, ethnicity and deprivation (unadjusted risk ratio (uRR) 1.00 (95\% CI 0.84 to 1.19 ), $\mathrm{p}=0.980$; adjusted risk ratio (aRR) 0.98 (95\% CI 0.83 to 1.17 ), $\mathrm{p}=0.844$ ). When patients' location, age, sex, ethnicity and deprivation were adjusted for, patients attending small practices had a $24 \%$ lower risk of hospital admission than patients attending large practices (aRR 0.76 (95\% CI 0.62 to 0.92$), p=0.006)$.

Patients admitted to hospital showed no difference in risk of hospital harm for rural compared with urban patients (uRR 1.17 (95\% CI 0.80 to 1.70 ), $\mathrm{p}=0.410$; aRR 1.01 (95\% CI 0.97 to 1.05$), p=0.587)$, as shown in table 3 . When the interactions between age and location (older urban people had greater risk of harm) and age and sex 
Table 3 Risk ratios for hospital harm: unadjusted and adjusted by location, age, sex, ethnicity, deprivation and general practice size

\begin{tabular}{|c|c|c|c|c|c|}
\hline & & Unadjusted risk ratio $(95 \% \mathrm{Cl})$ & P value* & Adjusted† risk ratio $(95 \% \mathrm{Cl})$ & P value* \\
\hline \multirow[t]{2}{*}{ Location } & Urban & Reference & 0.410 & Reference & 0.587 \\
\hline & Rural & 1.17 (0.80 to 1.70$)$ & & 1.01 (0.97 to 1.05$)$ & \\
\hline $\begin{array}{l}\text { Location-age } \\
\text { interaction }\end{array}$ & Per year & & & 0.98 (0.96 to 1.00$)$ & 0.030 \\
\hline Age & Per year & 1.02 (1.01 to 1.02$)$ & $<0.001$ & 1.02 (0.98 to 1.07$)$ & 0.322 \\
\hline $\mathrm{Age}^{2}$ & Per year $^{2}$ & & & 1.00 (1.00 to 1.00$)$ & 0.783 \\
\hline \multirow[t]{2}{*}{ Sex } & Male & Reference & 0.644 & Reference & 0.080 \\
\hline & Female & $1.08(0.76$ to 1.54$)$ & & 1.67 (0.94 to 2.96$)$ & \\
\hline Sex-age interaction & Per year & & & 1.01 (0.98 to 1.04$)$ & 0.572 \\
\hline Sex-age ${ }^{2}$ interaction & Per year ${ }^{2}$ & & & 1.000 (0.998 to 1.000$) \ddagger$ & 0.031 \\
\hline \multirow[t]{4}{*}{ Ethnicity } & European & Reference & 0.737 & Reference & 0.853 \\
\hline & Māori & $0.71(0.35$ to 1.44$)$ & & $0.76(0.37$ to 1.57$)$ & \\
\hline & Pacific & 0.69 (0.20 to 2.42$)$ & & $0.75(0.24$ to 2.31$)$ & \\
\hline & Other & 0.72 (021 to 2.47 ) & & 0.80 (0.26 to 2.46$)$ & \\
\hline \multirow{6}{*}{$\begin{array}{l}\text { Socioeconomic } \\
\text { status-NZDep }\end{array}$} & Quintile 1 & Reference & 0.244 & Reference & 0.160 \\
\hline & Quintile 2 & 1.12 (0.64 to 1.95$)$ & & 1.16 (0.66 to 2.03$)$ & \\
\hline & Quintile 3 & $1.40(0.74$ to 2.64$)$ & & 1.39 (0.68 to 2.85$)$ & \\
\hline & Quintile 4 & $1.58(0.80$ to $3 / 13)$ & & 1.77 (0.90 to 3.48$)$ & \\
\hline & Quintile 5 & 1.84 (0.97 to 3.51$)$ & & 1.90 (1.07 to 3.36$)$ & \\
\hline & No data & 2.24 (1.03 to 4.87$)$ & & 2.47 (1.08 to 5.65$)$ & \\
\hline \multirow[t]{3}{*}{ Practice size } & Large & Reference & 0.965 & Reference & 0.239 \\
\hline & Medium & 0.94 (0.58 to 1.51$)$ & & 0.73 (0.50 to 1.09$)$ & \\
\hline & Small & 0.97 (0.66 to 1.43$)$ & & 0.79 (0.55 to 1.13$)$ & \\
\hline
\end{tabular}

${ }^{*} \mathrm{P}$ values are from Poisson regression models with Wald tests used for categorical variables.

†Adjusted for all other variables in the table.

$\ddagger$ Three decimal places shown to indicate direction of effect.

NZ, New Zealand.

(older men had greater risk of harm) were included, the association seen between unadjusted age and greater risk of harm was no longer statistically significant.

There was no difference seen in the rate of hospital harm per admission comparing rural to urban patients, (uIRR 0.95 (95\% CI 0.62 to 1.46 ), $\mathrm{p}=0.822$, aIRR 1.09 (95\% CI 0.83 to 1.43 ), $\mathrm{p}=0.524$ ), as shown in table 4 . Socioeconomic status showed a significant overall association with rate of hospital harm per admission, but with no clear pattern of difference between rural and urban patients.

No difference between urban and rural patients was seen in the risk of experiencing preventable hospital harm (uRR 1.15 (95\% CI 0.83 to 1.60 ), $\mathrm{p}=0.679$; adjusted for age, sex and ethnicity aRR 1.12 (95\% CI 0.77 to $1.61), \mathrm{p}=0.550)$. No difference between rural and urban patients was seen in the proportional odds of being in higher harm severity categories (unadjusted proportional OR 0.76 (95\% CI 0.28 to 2.10), $\mathrm{p}=0.597$; proportional OR adjusted for age and sex 0.76 (95\% CI 0.28 to 2.03$)$, $\mathrm{p}=0.583$ ).

\section{Where hospital harm detected}

Hospital harm was detected during hospital admission or subsequent readmission in 55\% (95/172) of patients, as recorded in the general practice clinical record. For the remaining $45 \%(77 / 172)$ of patients, the hospital harm was identified only during general practice encounters, for example, postoperative infections treated in general practice. Rurality was not associated with this pattern, with hospital harm detected in the general practice record only for $43 \%$ of rural patients (40/93) and $47 \%$ of urban patients $(37 / 79)(\mathrm{p}=0.615)$.

\section{Interhospital transfer}

No urban patients required interhospital transfer in the study, but $3 \%$ of rural patients $(26 / 809)$ did. Table 5 shows rural patients' experience of hospital harm and interhospital transfer.

Patients requiring interhospital patient transfer had an associated more than doubled risk of hospital harm compared with patients not transferred (uRR 2.41 (95\% CI 1.54 to 3.77$), \mathrm{p}<0.001)$. This association persisted 
Table 4 Rate ratios of hospital harm per admission: unadjusted and adjusted by location, age, sex, ethnicity, deprivation and general practice size

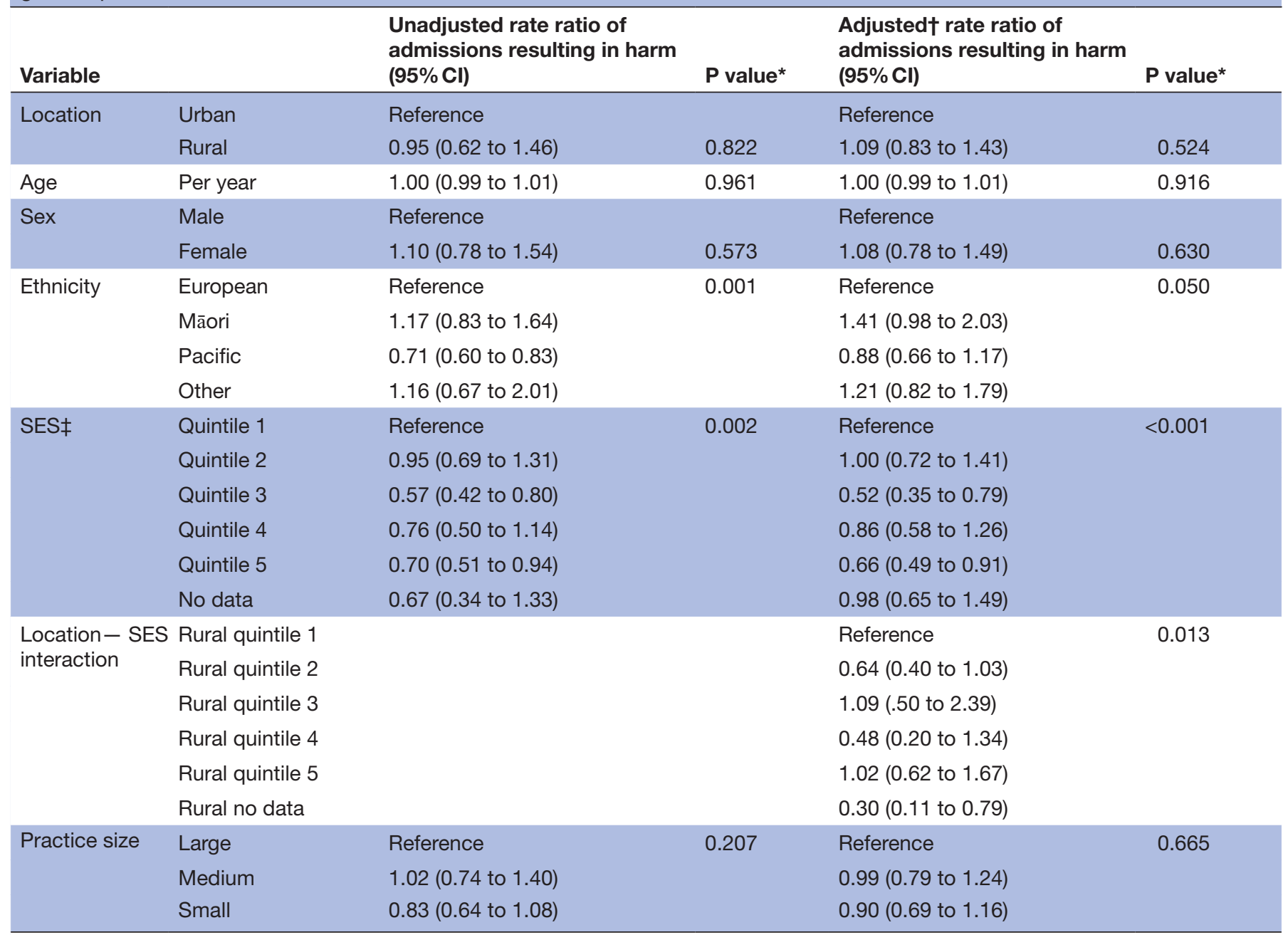

${ }^{*} \mathrm{P}$ values are from Poisson regression models, using Wald tests used for categorical variables.

†Adjusted for all other variables in the table.

$\ddagger$ Effects presented are fixed for 'urban' location.

SES, socioeconomic status.

when adjusted for age (aRR 2.33 (95\% CI 1.37 to 3.98), $\mathrm{p}=0.003)$ and separately for sex (aRR $2.41(95 \%$ CI 1.52 to 3.80$), \mathrm{p}=0.001)$. More detailed analysis was not possible given the small number (26) of patients transferred. ${ }^{26}$

Table 5 Patient interhospital transfer and hospital harm experienced by rural patients

\begin{tabular}{lcccc} 
& $\begin{array}{l}\text { Hospital } \\
\text { harm } \mathbf{n} \\
\text { All rural patients }\end{array}$ & $\begin{array}{l}\text { No hospital } \\
\text { harm } \mathbf{n} \text { (row } \\
\%)\end{array}$ & Total & P value* $^{*}$ \\
\hline $\begin{array}{l}\text { Interhospital } \\
\text { transfer }\end{array}$ & $7(27)$ & $19(73)$ & 26 & 0.022 \\
$\begin{array}{l}\text { No interhospital } \\
\text { transfer }\end{array}$ & $86(11)$ & $697(89)$ & 783 & \\
Total & $93(11)$ & $716(89)$ & 809 & \\
\hline
\end{tabular}

${ }^{*} \mathrm{P}$ value from $\chi^{2}$ test.

\section{DISCUSSION}

In our secondary analysis of a large retrospective NZ general practice records review study, we have found no significant difference in the risk of hospital admission or hospital harm experienced by patients attending rural and urban general practices. We did however identify that interhospital transfer was associated with a more than doubled risk of hospital harm for the small number of (only) rural patients requiring it. We showed that nearly half of all harms originating in hospital were identified only outside hospital settings in general practice. We also showed that patients enrolled in small general practices had a lower risk of hospital admission than patients enrolled in large practices.

\section{Strengths and limitations}

Secondary analysis of a large retrospective patient record review study has allowed us to investigate associations of 
clinical significance between patients' rural and urban location and hospital harm with mostly sufficiently precise estimates (as indicated by the $95 \%$ CIs), in a study designed to maximise statistical power to detect ruralurban differences. The random selection and high participation rate of practices in the original SHARP study ${ }^{19}$ would facilitate external validity. Our data on hospital admissions included private and public hospital admissions linked to patient-level demographics which should enhance the external validity of the results. By capturing patients' experiences of hospital harm using general practice electronic patient records, previously undetected harms have been identified. The weighted findings are likely to be generalisable to the wider NZ population, and to other countries with similar health systems and similar geographical contexts.

The original study group was stratified by rural-urban location of general practice and by general practice size. Known confounders of age, ethnicity and socioeconomic status ${ }^{28}$ were collected and adjusted for in statistical modelling. However, data on deprivation were not complete in $10 \%$ of records and this was twice as likely for rural patients. Addresses not recorded in an approved format cannot be geocoded, and no deprivation quintile can be assigned. Furthermore, sparsely populated mesh blocks (the geographical areas linked to deprivation levels) may not have deprivation data available to minimise data disclosure for residents. ${ }^{29}{ }^{30}$ Patients' location was defined by the general practice they were enrolled in, on the assumption that people attended general practices of the same urban or rural location as where they lived. Patients living in rural settings may choose to register at an urban general practice, underestimating the rurality effect in reported findings. The lack of standardised methods to record hospital admissions across contributing practices' clinical records meant that detection of hospital admissions is likely to have varied, and detected admission rates are likely to be an under-representation. A further limitation is that data on patient health or illness status were not collected and are likely to be residual confounders of these results. People from rural communities may migrate to urban settings as they became more unwell, noting that frail elderly people with multiple comorbidities are more likely to experience hospital harm. ${ }^{31}$ Rural patients may delay seeking care ${ }^{32}$ and may be more unwell when admitted to hospital with reduced physical reserve, making them more susceptible to harm.

\section{Hospital harm and patient rurality}

The limited research available into differences in hospital related patient safety by rural-urban location comes from the USA and relates to the location of hospitals. A 2004 literature review concluded that while there was not enough published information to be definitive, rates of adverse events in rural hospitals appeared to be no worse than in urban hospitals, nor in smaller ( $<50$ beds) rural hospitals compared with larger ( $>100$ beds) rural hospitals. ${ }^{9}$ A 2010 comparison of patient safety outcome indicators found the quality of care provided in small rural hospitals was the same as in small urban hospitals in 292 American hospitals with $<100$ beds. ${ }^{8}$ In our study, we used the rural and urban location of the general practice patients attended as a proxy for rural and urban place of residence. We could find no other published research investigating hospital harm using patients' place of residence. Our findings of no evidence for differences in hospital harm, preventable harm or severity of harm for people living in rural and urban settings is reassuring for rural communities and health planners, although the 95\% CIs of our findings included some values that would be clinically significant.

The only area of difference was seen in interhospital transfer. All interhospital transfers in the study data occurred in rural patients, and the $3 \%$ of rural patients requiring interhospital patient transfer had an associated more than doubled risk of hospital harm. While there is no previous published NZ data on interhospital transfer rates, research from 2002/3 indicated that there was a wide range in rural hospitals' ability to treat presenting conditions without need to transfer patients. ${ }^{33} \mathrm{NZ}$ clinical practice has changed considerably in the last decade with the advent of rural hospital medicine specialists working in rural hospitals. ${ }^{34}$ International studies reports that rural patients have higher rates of transfer than urban patients, ${ }^{11}$ with higher rates of adverse outcomes as noted above. ${ }^{10-13}$ Some studies show this excess in adverse outcomes persists when patient characteristics and underlying illnesses are controlled for. ${ }^{12}{ }^{13}$ Poorer outcomes for transferred patients come from a combination of patient factors and system factors relating to the processes of transfer. Patients who are transferred between hospitals are vulnerable to adverse outcomes due to the complex and unstable medical problems necessitating transfer. System issues of poor communication and handover between referring, transferring and receiving clinical teams, ${ }^{12} 143536$ and limited resources and space during transfers to monitor and address evolving changes in health status ${ }^{1214}$ are also implicated. Our data did not include information on patient health status and we could not explore the association between interhospital transfer and patient harm further.

\section{The true burden of hospital harms}

Our study indicated that $11 \%$ of patients admitted to hospital experienced hospital harm. A systematic review of hospital patient record review studies showed that approximately $9 \%$ of patients admitted to hospital experience adverse events, noting differences in the data collection process between countries. ${ }^{16}$ Just under half of hospital harms in our study did not become apparent until after discharge and did not trigger re-admission, so would not be detected using hospital records review study methodology. ${ }^{1637}$ While these extra harms we have detected did not trigger a readmission they impacted on patients, for example, causing prolonged return to normal work and home life. Hospital harms recorded in 
discharge summary correspondence sent to general practice are likely to exclude more minor inpatient harms, such as one-off medication dispensing errors with no lasting patient impact. It is therefore likely that the 'true' level of patient harm relating to hospital admission is a combination of harms detected in traditional hospitalbased studies, and harms occurring in the community that are dealt with by general practice without further hospital contact. Including general practice data captures a more complete picture of hospital-related harm than hospital records alone can provide.

\section{General practice size and hospital admissions}

Patients enrolled in small practices had a $24 \%$ lower adjusted risk of hospital admission over the 3-year period than patients enrolled in large practices. Analysis of the characteristics of the general practices of the original SHARP study showed that large practices had significantly higher numbers of patients enrolled per fulltime-equivalent (FTE) general practitioner (GP) (mean 1827 patients per FTE GP) compared with medium-sized practices (mean 1457 patients/FTE GP) and small practices (mean 1120 patients/FTE GP) but similar practice nurse workloads. ${ }^{24}$ International literature on the linkage between practice size, patient caseload for the general practice team and quality and continuity of primary care is mixed. An English study of over 230000 patients showed that better continuity of care with one's own GP was associated with reduced rates of preventable hospital admissions and that larger practices ( $>7$ FTE GPs) had lower levels of continuity than smaller practices (1-3 FTE GPs).$^{38}$ A systematic review of the effect of practice size on quality of primary care found that there was limited evidence to support a link between size and quality, with different attributes of quality favouring larger or smaller practices. ${ }^{39}$ Another systematic review showed that greater continuity of care by generalist (and specialist) doctors was associated with reduced mortality rates. Whether our findings reflect smaller practices with lower patient to GP ratio contributing to greater continuity of care with less unnecessary patient admissions is not clear, as the type of hospital admission (planned or unplanned) is not captured in the dataset here, and the literature to support such a theory is inconclusive.

\section{Implications for policy and practice}

The study's finding of no evidence of differences in hospital harm by patient location provides reassurance to rural communities and health planners that current harm minimisation strategies appear to provide comparable care for people living in rural and urban settings. This suggests that greater centralisation of hospital level services in the NZ context is not necessary from a patient safety perspective. The association seen between interhospital transfer and more than double the risk of hospital harm for the small number of rural patients requiring transfer warrants further attention. Given international trends towards greater use of telehealth in smaller sites and centralisation of health services, ${ }^{40}$ more research to understand the contribution of interhospital transfer to patient harm and the role of patient factors and system factors in those poorer outcomes is needed. This should include larger samples of patients than in this study to confirm the association, and collect patient-level data on coexisting health problems and severity of current illness as well as transfer, communication and handover processes between different healthcare providers caring for transferred patients.

The findings of an association between smaller practice size and lower risk of hospital admission raises questions about potential benefits of smaller general practices. This is juxtaposed against the trend towards consolidation of general practice services into larger provider units to allow subspecialisation within general practice and colocation and integration with other health services. ${ }^{41}$ Further research to explore associations between practice size and hospital usage is warranted, including investigating possible mediators of any association, such as care continuity, using datasets that can account for potential confounders such as patient comorbidity and organisational structure. The findings that nearly half of all harms originating in hospital settings became apparent only in the general practice setting indicates that general practice clinical data should be included when examining the full burden of hospital harm.

\section{CONCLUSION}

Our study has shown no evidence of a difference in hospital harm experienced by people living in rural and urban settings, which provides comfort for rural communities and health planners. The exception is when interhospital transfer is required, where the risk is more than doubled. Further investigation is needed to understand if this is solely related to patients being more unwell when needing transfer, or if the transfer process itself increases risk of harm.

\section{Twitter Carol Atmore @drcarolatmore and Tim Stokes @StokesTim63}

Acknowledgements The authors thank the Safety, Harms and Risk reduction Project study team, without whom this research could not have proceeded (S Dovey, K Wallis, M Williamson, W Cunningham, S Lillis, D Reith, M Tilyard, K Eggleton and A McMenamin) and the participating study practices; and the Health Research Council of New Zealand and the University of Otago for funding this research.

Contributors CA, SD and RG designed the study and developed the study protocol. $\mathrm{CA}$ undertook the clinical record reviews to collect the data for analysis. CA and ARG performed statistical analyses and interpreted the analytical results. TS, SD, RG and ARG supervised the study. CA wrote the manuscript. All authors made critical revisions and approved the final version of the manuscript.

Funding This study was funded by the Health Research Council of New Zealand, Foxley Fellowship grant HRC16/056, and by the Dunedin School of Medicine, Otago Medical School, University of Otago through a Clinical Research Scholarship.

Disclaimer The funding bodies had no involvement in the design of the study and collection, analysis and interpretation of data and in writing the manuscript

Competing interests None declared.

Patient and public involvement Patients and/or the public were not involved in the design, or conduct, or reporting, or dissemination plans of this research. 
Patient consent for publication Not required.

Ethics approval Ethics approval was gained from the Human Ethics Committee, University of Otago, 16/084.

Provenance and peer review Not commissioned; externally peer reviewed.

Data availability statement No data are available. Due to the sensitive nature of the research topic, it was undertaken to keep the data confidential to the research team, and the general practices consented to participate in the study on that basis.

Open access This is an open access article distributed in accordance with the Creative Commons Attribution Non Commercial (CC BY-NC 4.0) license, which permits others to distribute, remix, adapt, build upon this work non-commercially, and license their derivative works on different terms, provided the original work is properly cited, appropriate credit is given, any changes made indicated, and the use is non-commercial. See: http://creativecommons.org/licenses/by-nc/4.0/.

\section{ORCID iDs}

Carol Atmore http://orcid.org/0000-0002-4031-7016

Robin Gauld http://orcid.org/0000-0001-5401-1192

Andrew R Gray http://orcid.org/0000-0003-4299-2194

Tim Stokes http://orcid.org/0000-0002-1127-1952

\section{REFERENCES}

1 Brezzi M, Dijkstra L, Ruiz V. OECD extended regional typology: the economic performance of remote rural regions. OECD regional development working papers. Paris: OECD, 2011.

2 Stats NZ. Subnational population estimates (urban rural), by age and sex, at 30 June 1996, 2001, 2006-18 (2018 boundaries) Wellington: STATs NZ, 2019. Available: http://nzdotstat.stats.govt.nz/wbos/ [Accessed 7 May 2019].

3 Atmore C. Guest Editorial: providing health care across distanceScottish solutions to rural challenges. J Prim Health Care 2016;8:189-92.

4 Prior M, Farmer J, Godden DJ, et al. More than health: the added value of health services in remote Scotland and Australia. Health Place 2010;16:1136-44.

5 Davidson D, Ellis Paine A, Glasby J, et al. Analysis of the profile, characteristics, patient experience and community value of community hospitals: a multimethod study. Health Serv Deliv Res 2019;7:1-152.

6 Moscovice I, Rosenblatt R. Quality-of-care challenges for rural health. J Rural Health 2000;16:168-76.

7 Institute of Medicine. Quality through collaboration: the future of rural health care. Washington, DC: The National Academies Press, 2004.

8 Vartak S, Ward MM, Vaughn TE. Patient safety outcomes in small urban and small rural hospitals. J Rural Health 2010;26:58-66.

9 Coburn AF, Wakefield M, Casey M, et al. Assuring rural hospital patient safety: what should be the priorities? J Rural Health 2004;20:314-26

10 Hinde YR, Pennington R, Nott ML. Time to surgery for hip fracture patients in a rural orthopaedic referral hospital. Aust $J$ Rural Health 2017;25:42-4.

11 Erickson T, Vana PG, Blanco BA, et al. Impact of hospital transfer on surgical outcomes of intestinal atresia. Am J Surg 2017;213:516-20.

12 Sokol-Hessner L, White AA, Davis KF, et al. Interhospital transfer patients discharged by academic hospitalists and general internists: characteristics and outcomes. J Hosp Med 2016;11:245-50.

13 Mohr NM, Harland KK, Shane DM, et al. Inter-hospital transfer is associated with increased mortality and costs in severe sepsis and septic shock: an instrumental variables approach. J Crit Care 2016;36:187-94.

14 Lyphout C, Bergs J, Stockman W, et al. Patient safety incidents during interhospital transport of patients: a prospective analysis. Int Emerg Nurs 2018;36:22-6.

15 Davis P, Lay-Yee R, Briant R, et al. Adverse events in New Zealand public hospitals I: occurrence and impact. N Z Med J 2002;115:271-80.

16 de Vries EN, Ramrattan MA, Smorenburg SM, et al. The incidence and nature of in-hospital adverse events: a systematic review. Qual Saf Health Care 2008;17:216-23.

17 Classen DC, Resar R, Griffin F, et al. Global trigger tool' shows that adverse events in hospitals may be ten times greater than previously measured. Health Aff 2011;30:581-9.
18 Runciman W, Hibbert P, Thomson R, et al. Towards an international classification for patient safety: key concepts and terms. Int J Qual Health Care 2009;21:18-26.

19 Dovey SM, Leitch S, Wallis KA, et al. Epidemiology of patient harms in New Zealand: protocol of a general practice records review study. JMIR Res Protoc 2017;6:e10.

20 Zegers M, de Bruijne MC, Wagner C, et al. The inter-rater agreement of retrospective assessments of adverse events does not improve with two reviewers per patient record. J Clin Epidemiol 2010;63:94-102.

21 Gisev N, Bell JS, Chen TF. Interrater agreement and interrater reliability: key concepts, approaches, and applications. Res Social Adm Pharm 2013;9:330-8.

22 Hanskamp-Sebregts M, Zegers M, Vincent C, et al. Measurement of patient safety: a systematic review of the reliability and validity of adverse event detection with record review. BMJ Open 2016;6:e011078.

23 Statistics New Zealand. New Zealand: an urban/rural profile. Wellington: Statistics New Zealand, 2005.

24 Leitch S, Dovey SM, Samaranayaka A, et al. Characteristics of a stratified random sample of New Zealand general practices. J Prim Health Care 2018;10:114-24.

25 Rogers W. Regression standard errors in clustered samples. Stata technical bulletin 1994;3.

26 Peduzzi P, Concato J, Feinstein AR, et al. Importance of events per independent variable in proportional hazards regression analysis. II. accuracy and precision of regression estimates. J Clin Epidemiol 1995;48:1503-10.

27 StataCorp L. Stata statistical software: release 15 College Station, tx 2017.

28 Smith KB, Humphreys JS, Wilson MGA. Addressing the health disadvantage of rural populations: how does epidemiological evidence inform rural health policies and research? Aust J Rural Health 2008:16:56-66.

29 Ministry of Health. Addressing and geocoding information for health providers. Wellington: Ministry of Health, 2017. https://www. health.govt.nz/our-work/health-identity/addressing-and-geocoding/ addressing-and-geocoding-information-health-providers

30 Garces-Ozanne A, Yow A, Audas R. Rural practice and retention in New Zealand: an examination of New Zealand-trained and foreigntrained doctors. N Z Med J 2011;124:14-23.

31 Hogan H, Healey F, Neale G, et al. Preventable deaths due to problems in care in English acute hospitals: a retrospective case record review study. BMJ Qual Saf 2012;21:737-45.

32 Brundisini F, Giacomini M, DeJean D, et al. Chronic disease patients experiences with accessing health care in rural and remote areas: a systematic review and qualitative meta-synthesis. Ont Health Technol Assess Ser 2013;13:1-33.

33 Williamson M, Gormley A, Dovey S, et al. Rural hospitals in New Zealand: results from a survey. N Z Med J 2010;123:20-9.

34 Nixon G, Blattner K, NZ Rural Hospital Doctors Working Party. Rural hospital medicine in New Zealand: vocational registration and the recognition of a new scope of practice. N Z Med J 2007;120:U2654.

35 Bradley NL, Garraway N, Bell N, et al. Data capture and communication during transfers to definitive care in an inclusive trauma system. Injury 2017;48:1069-73.

36 Piper D, Lea J, Woods C, et al. The impact of patient safety culture on handover in rural health facilities. BMC Health Serv Res 2018; 18:889.

37 Davis P, Lay Yee R, Briant R. Adverse events in New Zealand public hospitals: principal findings from a national survey. Wellington: Ministry of Health, 2001.

38 Barker I, Steventon A, Deeny SR. Association between continuity of care in general practice and hospital admissions for ambulatory care sensitive conditions: cross sectional study of routinely collected, person level data. BMJ 2017;356:j84.

$39 \mathrm{Ng}$ CWL, $\mathrm{Ng}$ KP. Does practice size matter? review of effects on quality of care in primary care. Br J Gen Pract 2013;63:e604-10.

40 Rechel B, Džakula A, Duran A, et al. Hospitals in rural or remote areas: an exploratory review of policies in 8 high-income countries. Health Policy 2016;120:758-69.

41 Charles A. Integrated care systems explained: making sense of systems places and neighbourhoods. London: The Kings Fund, 2020.

42 Ministry of Health. Ethnicity data protocols for the health and disability sector. Wellington, New Zealand: Ministry of Health, 2004.

43 Atkinson J, Salmond C, Crampton P. NZDep2013 index of deprivation. Wellington: Department of Public Health, University of Otago, 2014. 